\title{
Peroxiredoxins as Markers of Oxidative Stress in IgA Nephropathy, Membranous Nephropathy and Lupus Nephritis
}

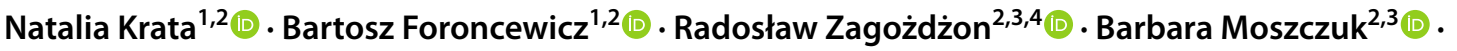 \\ Magdalena Zielenkiewicz ${ }^{5}$ (D) . Leszek Pączek ${ }^{1,2,4}$ (D) Krzysztof Mucha ${ }^{1,2,4} \mathbb{D}$
}

Received: 30 July 2021 / Accepted: 12 November 2021 / Published online: 16 December 2021

(c) The Author(s) 2021

\begin{abstract}
IgA nephropathy (IgAN), membranous nephropathy (MN), and lupus nephritis (LN) represent important causes of chronic kidney disease. They belong to the immune-mediated glomerulonephritis (GNs), and have distinct pathogenesis, distinct clinical courses, and variable responses to treatment. Therefore, specific diagnostic procedures are necessary for more effective patient management. Recently, a role for oxidative stress has been proposed in various renal disorders. Thus, molecules related to oxidative stress, such as 2-Cys-peroxiredoxins (PRDXs), may represent plausible candidates for biomarkers in renal pathologies. The aim of this study was to assess whether there are differences between individual GNs and healthy controls in the context of PRDXs serum concentration. We enrolled 108 patients with biopsy-proven IgAN (47), MN (26), LN (35) and 30 healthy age- and sex-matched controls. The serum concentrations of PRDX 1-5 were measured with ELISA assays and correlated with demographic and clinical data. The PRDXs' concentration varied depending on the GN type. We also observed an association of PRDXs with lower estimated glomerular filtration rates, complement, hemoglobin, and body mass index. Our study indicates that individual PRDX can play roles in pathophysiology of selected GNs and that their serum concentrations may become useful as a new supplementary diagnostic markers in IgAN, MN as well as LN. The results of this study open a new avenue for prospective research on PRDXs in renal diseases.
\end{abstract}

Keywords Chronic kidney disease $\cdot \operatorname{IgA}$ nephropathy $\cdot$ Lupus nephritis $\cdot$ Membranous nephropathy $\cdot$ Oxidative stress . Peroxiredoxins

Krzysztof Mucha

kmucha@wum.edu.pl;kjmucha@gmail.com

1 Department of Immunology, Transplantology and Internal Diseases, Medical University of Warsaw, Warsaw, Poland

2 ProMix Center (ProteogenOmix in Medicine) at the Department of Immunology, Transplantology and Internal Diseases, Medical University of Warsaw, Warsaw, Poland

3 Department of Clinical Immunology, Medical University of Warsaw, Warsaw, Poland

4 Institute of Biochemistry and Biophysics, Polish Academy of Sciences, Warsaw, Poland

5 Faculty of Mathematics, Informatics and Mechanics, University of Warsaw, Warsaw, Poland

\section{Introduction}

Chronic kidney disease (CKD) is a growing public health problem, affecting approximately $8-13 \%$ of the population (Brück et al. 2016; Hill et al. 2016). Furthermore, it is projected that in 2040, CKD will be the fifth leading cause of death in the world (Foreman et al. 2018). Glomerulonephropathies (GNs) such as IgA nephropathy (IgAN), membranous nephropathy (MN), and lupus nephritis (LN) are immune-mediated, although they have different etiologies, and are among the most frequent causes of CKD (Pippias et al. 2017). GNs account for about $20 \%$ of CKD cases in most countries, usually affecting young people and carry a lifelong CKD burden (Floege and Amann 2016). IgAN and $\mathrm{MN}$ belong to the primary GNs, and their annual incidence is estimated at $2-5$ and $1-2$ cases per 100,000 adults, respectively (McGrogan et al. 2011). LN develops secondary to systemic disease, and its incidence is estimated at $0.4-0.7$ cases per 100,000 population per year (Patel et al. 2006). 
Unfortunately, GNs frequently progress asymptomatically or present with proteinuria, erythrocyturia or hematuria, edema, and hypertension (Vassalotti et al. 2010). These symptoms are neither specific nor sensitive enough for any GN. The diagnosis is difficult and frequently too late, and still requires histopathological evaluation by kidney biopsy, an invasive procedure with known risks (Mucha et al. 2016). Therefore, alternative, specific, reproducible, and safer methods are needed to facilitate noninvasive diagnosis.

There are specific markers that may help to diagnose glomerular diseases, including galactose-deficient IgA1 and IgG autoantibodies that correlate with IgAN (Placzek et al. 2018), anti-phospholipid 2 receptor antibodies that correlate with the histological picture of MN, and anti-double-stranded DNA antibodies associated with LN activity ( $\mathrm{Na}$ et al. 2017). Multiple urine and serum proteins (Gao et al. 2018; Krata et al. 2018; Moszczuk et al. 2021; Mucha et al. 2014) or gene polymorphisms (Xie et al. 2020; Pac et al 2021) have been proposed as markers of different kidney diseases in the last decade. However, their diagnostic and/or prognostic utility remains to be validated (Krata et al. 2018; Selvaskandan et al. 2020; Sethi et al. 2019, 2020; Yanagawa et al. 2014). In this study, we focused on oxidative stress-related markers, 2-cysteine peroxiredoxins (2-Cys PRDXs), as potentially discriminatory in renal diseases.

Oxidative stress (OS) is one of the mechanisms involved in the progression of every type of CKD, including GN (Krata et al. 2018). Indeed, specific CKD-related conditions may lead to the overproduction of reactive oxygen species (ROS). It was reported that CKD patients have increased levels of plasma thiol oxidation and carbonylation, but the role of PRDXs in the pathophysiology of kidney diseases remains unknown (Cachofeiro et al. 2008; Krata et al. 2018). PRDXs, which are similar in function to well-known antioxidant enzymes such as catalase and glutathione peroxidase, possess the ability to reduce excessive levels of hydrogen peroxide, one of the major OS mediators (Jeong et al. 2012; Yang and Lee 2015). Importantly, kinetics measurements imply that PRDXs reduce more than $90 \%$ of cellular peroxides (Adimora et al. 2010; Perkins et al. 2015; Winterbourn 2008), which predisposes them to being a crucial factor in cellular OS regulation. Oxidative stress has been reported in kidney disease, due to both antioxidant depletions as well as increased production of ROS (Daenen et al. 2019; Irazabal and Torres 2020). The kidney is a highly metabolic organ, rich in oxidation reactions in mitochondria, which makes it vulnerable to damage caused by ROS (Aranda-Rivera et al. 2021). Therefore, OS can accelerate kidney disease progression. Different PRDX isoforms were reported to be involved in diabetic nephropathy (Lee and Lee 2018), ischemia/reperfusion damage (Sharapov et al. 2020), obstructive kidney disease (Hwang et al. 2019), ciliopathies (Zacchia et al. 2020) and acute tubular necrosis (Wu et al. 2017). However, the role of PRDXs in the pathophysiology of glomerular diseases is not well known.

In the current study, we hypothesized that 2-Cys PRDXs could be differentially involved in IgAN, MN, and LN. If so, the PRDX family could serve as additional markers of specific GNs. Therefore, the aim of this study was to evaluate PRDX 1-5 serum concentrations in IgAN, $\mathrm{MN}$, and LN patients and healthy controls.

\section{Materials and Methods}

\section{Patients}

We enrolled 108 patients (GN group) diagnosed by renal biopsy with IgAN (47), MN (26), and LN (35). The exclusion criteria were active infection, current pregnancy, history of malignancy, or previous organ transplantation. The healthy control group was defined by the absence of any kidney disease or other chronic diseases requiring treatment and consisted of 30 age- and sex-matched volunteers. Demographic characteristics of study participants are presented in Table 1. The study was performed in accordance with the Declaration of Helsinki guidelines for research on human subjects and was approved by the Ethics Committee of the Medical University of Warsaw (KB/9/2010 and KB/199/2016), and written informed consent was obtained from all the participants.

\section{Methods}

\section{Material Collection}

Blood samples were collected once from each of the individuals (fasting) into serum separating tubes (Becton Dickinson, Franklin Lakes, NJ, USA). To obtain serum, blood samples were left to clot at $23-25{ }^{\circ} \mathrm{C}$ (room temperature, RT) for $30 \mathrm{~min}$ and centrifuged at $2000 \mathrm{RPM}$ at RT. Serum samples were stored in aliquots at $-80{ }^{\circ} \mathrm{C}$ until further measurements.

\section{PRDX Measurements}

The serum concentration of each of PRDX (1-5) was measured using commercially available enzyme-linked immunosorbent assays (EIAab, Wuhan, China). Briefly, the samples and standards were added to the microtiter plate and pre-coated with a biotin-conjugated antibody specific to the target antigen. The standards and samples 
Table 1 Characteristics of study participants

\begin{tabular}{|c|c|c|c|c|c|c|}
\hline & Healthy controls & $\mathrm{IgAN}$ & $\mathrm{MN}$ & $\mathrm{LN}$ & GN group & $P$ value \\
\hline \multicolumn{7}{|l|}{ Demographics } \\
\hline Age (years) & $44.4 \pm(12.9)$ & $43.1 \pm(13.3)$ & $54.1 \pm(13.4)$ & $45.2 \pm(12.4)$ & $46.4 \pm(13.6)$ & 0.022 \\
\hline Male $(\%) * *$ & $43.3 \%$ & $51.1 \%$ & $57.7 \%$ & $31.4 \%$ & $46.3 \%$ & 0.773 \\
\hline $\operatorname{BMI}\left(\mathrm{kg} / \mathrm{m}^{2}\right)$ & $23.8 \pm(3.4)$ & $27.3 \pm(3.8)$ & $25.7 \pm(4.1)$ & $24.7 \pm(7.9)$ & $26.1 \pm(5.5)$ & $<0.001$ \\
\hline \multicolumn{7}{|l|}{ Laboratory data } \\
\hline WBC (G/L) & $5.6 \pm(1.02)$ & $8.2 \pm(2.4)$ & $8.6 \pm(3.1)$ & $7.0 \pm(3.5)$ & $7.9 \pm(3.02)$ & $<0.001$ \\
\hline $\mathrm{HGB}(\mathrm{g} / \mathrm{dL})$ & $13.9 \pm(1.2)$ & $14.1 \pm(1.5)$ & $13.6 \pm(1.8)$ & $13.2 \pm(1.8)$ & $13.5 \pm(2.4)$ & 0.128 \\
\hline $\mathrm{HCT}(\mathrm{L} / \mathrm{L})$ & $0.422 \pm(0.036)$ & $0.434 \pm(0.042)$ & $0.423 \pm(0.052)$ & $0.411 \pm(0.056)$ & $0.424 \pm(0.049)$ & 0.289 \\
\hline PLT (G/L) & $236.2 \pm(53.8)$ & $251.3 \pm(54.6)$ & $248.5 \pm(78.9)$ & $243.6 \pm(87.2)$ & $248.1 \pm(71.5)$ & 0.842 \\
\hline C3 $(\mathrm{mg} / \mathrm{dL})^{*}$ & n.a & $114.1 \pm(27.7)$ & $106.5 \pm(48.9)$ & $94 \pm(26.6)$ & $106.1 \pm(30.2)$ & 0.006 \\
\hline $\mathrm{C} 4(\mathrm{mg} / \mathrm{dL}) *$ & n.a & $26.2 \pm(6.9)$ & $29.0 \pm(11.3)$ & $16.3 \pm(8.0)$ & $22.8 \pm(9.0)$ & $<0.001$ \\
\hline Serum creatinine $(\mathrm{mg} / \mathrm{dL})$ & $0.86 \pm(0.14)$ & $1.47 \pm(1.11)$ & $1.13 \pm(0.46)$ & $1.07 \pm(0.68)$ & $1.26 \pm(0.87)$ & 0.013 \\
\hline eGFR $\left(\mathrm{ml} / \mathrm{min} / 1.73 \mathrm{~m}^{2}\right)$ & $92.2 \pm(13.7)$ & $71.7 \pm(34.4)$ & $74.2 \pm(26.2)$ & $82.1 \pm(27.9)$ & $75.6 \pm(30.5)$ & 0.059 \\
\hline $\begin{array}{l}\text { Proteinuria* } \\
\text { (g/24 h) }\end{array}$ & n.a & $0.96 \pm(1.14)$ & $1.00 \pm(1.23)$ & $0.46 \pm(0.63)$ & $0.82 \pm(1.05)$ & $<0.001$ \\
\hline Total serum protein $(\mathrm{g} / \mathrm{dL})$ & $7.4 \pm(0.3)$ & $7.0 \pm(0.6)$ & $6.5 \pm(1.2)$ & $7.0 \pm(0.5)$ & $6.9 \pm(0.7)$ & 0.139 \\
\hline Serum albumin $(g / d L)$ & $4.3 \pm(0.1)$ & $3.9 \pm(0.4)$ & $3.9 \pm(0.6)$ & $3.9 \pm(0.3)$ & $3.9 \pm(0.4)$ & 0.029 \\
\hline Serum $\alpha-1(\mathrm{~g} / \mathrm{dL})$ & $0.3 \pm(0.03)$ & $0.3 \pm(0.05)$ & $0.3 \pm(0.06)$ & $0.3 \pm(0.06)$ & $0.3 \pm(0.05)$ & 0.345 \\
\hline Serum $\alpha-2(g / d L)$ & $0.6 \pm(0.07)$ & $0.725 \pm(0.1)$ & $0.8 \pm(0.12)$ & $0.789 \pm(0.13)$ & $0.750 \pm(0.11)$ & 0.011 \\
\hline Serum $\beta-1(\mathrm{~g} / \mathrm{dL})$ & $0.5 \pm(0.05)$ & $0.487 \pm(0.07)$ & $0.477 \pm(0.07)$ & $0.478 \pm(0.11)$ & $0.484 \pm(0.07)$ & 0.909 \\
\hline Serum $\beta-2(g / d L)$ & $5.6 \pm(1.4)$ & $4.7 \pm(2.8)$ & $6.0 \pm(1.2)$ & $4.9 \pm(2.6)$ & $5.00 \pm(2.6)$ & 0.413 \\
\hline Serum $\gamma(\mathrm{g} / \mathrm{dL})$ & $1.3 \pm(0.2)$ & $1.1 \pm(0.2)$ & $0.8 \pm(0.3)$ & $1.1 \pm(0.3)$ & $1.1 \pm(0.3)$ & 0.018 \\
\hline
\end{tabular}

Values are given as mean $\pm \mathrm{SD}$. Level of significance was calculated with Chi-squared test and non-parametric Kruskal-Wallis test. $P<0.05$ indicates that at least one studied group is significantly different from one other group. *Healthy controls group excluded from comparison; **comparison between GN and healthy control; n.a. not available; $B M I$ body mass index, $W B C$ white blood count, $H G B$ hemoglobin, $H C T$ hematocrit, $P L T$ platelets, $e G F R$ estimated glomerular filtration rate

were added in a determined order the amount of $100 \mu \mathrm{L}$ per well. Then, avidin-conjugated horseradish peroxidase was added to each microplate well. The enzyme-substrate reaction was terminated by the addition of sulfuric acid solution. Color changes in each well were measured spectrophotometrically at a wavelength of $450 \mathrm{~nm}$ on a BioTekPowerWave XS microplate reader (BioTek, Winooski, VT, USA). The targeted antigen concentration was determined by comparing the optical density absorbance of samples to the standard curve. Samples below the detection range for each test were set as the lowest concentration obtained from the standard curve to avoid losing the meaningful part of the results and compute reliable statistical analysis.

\section{Biochemical and Clinical Characteristics}

Laboratory tests of serum creatinine, proteins, complement, blood morphology, urine analysis, and urinary protein were assayed by routine laboratory techniques. The estimated glomerular filtration rate (eGFR) was calculated according to the Chronic Kidney Disease-Epidemiology Collaboration equation. Body weight in kilograms was divided by the square of height in meters $\left(\mathrm{kg} / \mathrm{m}^{2}\right)$ to evaluate body mass index (BMI).

\section{Statistical Analysis}

Statistical analysis was performed in $\mathrm{R}$ version 3.6.1. and Statistica 13.1 (StatSoft). Results were expressed as mean \pm standard deviation, median \pm interquartile range, or a percentage value. All variables were tested for normal distribution by the Shapiro-Wilk test. Non-normally distributed variables were analyzed by non-parametric tests. Comparisons between demographic data were tested by the Kruskal-Wallis test (quantitative variables) and Chi-squared test (qualitative variables), whereas comparisons in biomarker levels between control and GN groups were tested by the Mann-Whitney $U$ test. Given that the biomarkers are non-normally distributed, the association between pairs of parameters were analyzed using Spearman's correlation. To correct for testing multiple hypotheses, in PRDX assessment in three GN groups, we used the Bonferroni method - given a total number of 15 comparative tests: 5 biomarkers and 3 disease groups compared to controls, we considered $P$ value $<0.05 / 15$ or 0.0033 

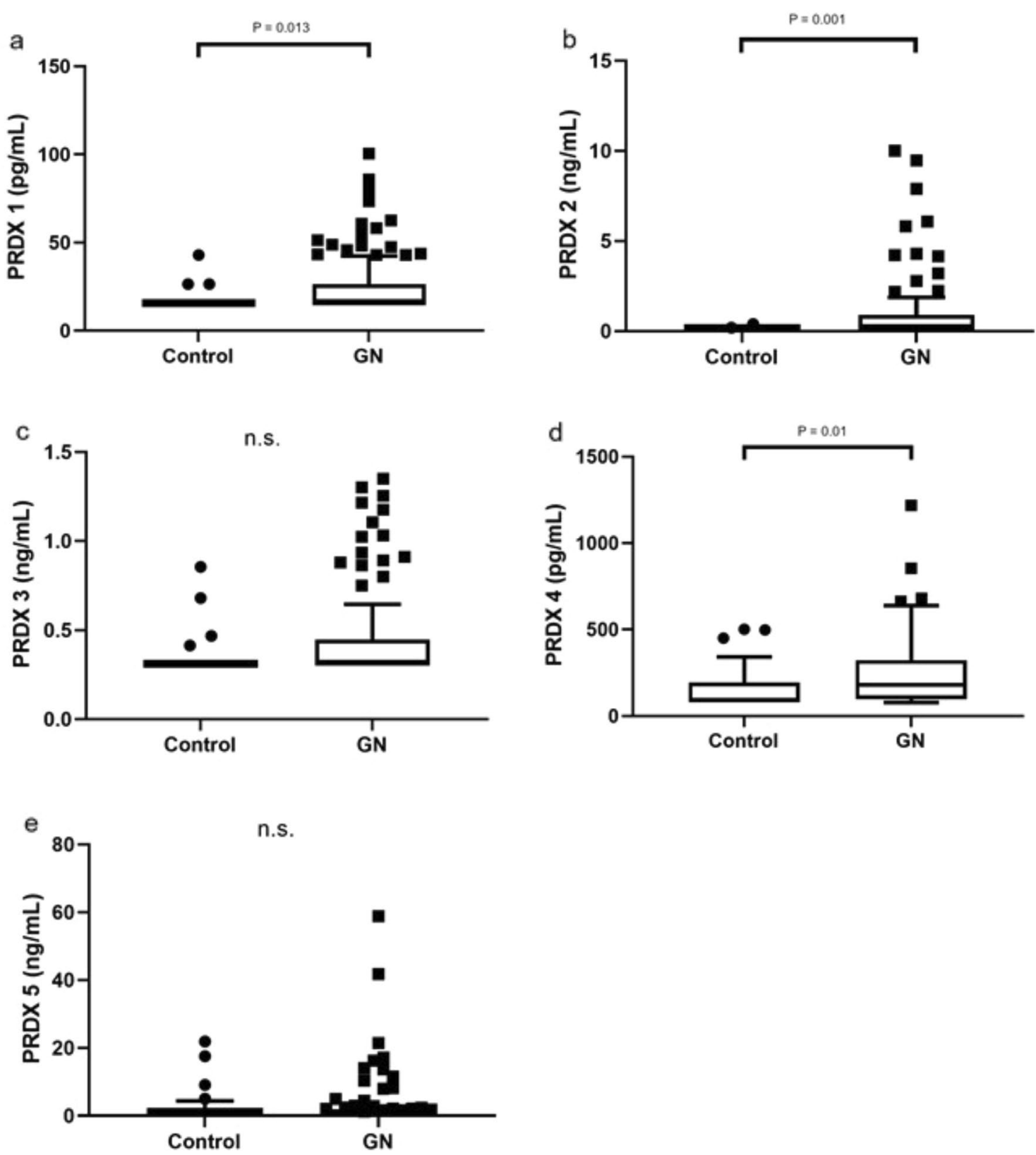

Fig. 1 PRDX 1-5 concentrations in patients with GN and healthy controls. Data are presented as box-and-whisker plots; box represents interquartile range (IRQ) with line set as median value for each PRDX concentration, ends of whiskers represent \pm 1.5 IQR of value

statistically significant. Receiver-operating characteristic curves (ROC) were calculated with cutoff points established by binary logistic regression with a significance level of $P<0.05$ and $95 \%$ confidence interval. The ROC analysis results were interpreted as follows: $\mathrm{AUC}<0.50$, low diagnostic accuracy; AUC in the range of 0.50-0.70, moderate diagnostic accuracy; and AUC $>0.70$, high diagnostic accuracy.

(maximum/minimum), and individual data points indicate outliers. $P<0.05$ was considered significant (Mann-Whitney $U$ test). GNIgAN, MN, LN combined; (a) PRDX 1; (b) PRDX 2; (c) PRDX 3; (d) PRDX 4; (e) PRDX 5; n.s.- - not significant

\section{Results}

\section{Discrimination Between GN Patients and Healthy Subjects}

We were able to discriminate GN patients in total from healthy subjects based on significantly elevated PRDX 1, 2, and 4 (Fig. 1a, b, d). No differences in PRDX 3 and 5 levels were observed between GN and controls (Fig. 1c, e); however, 

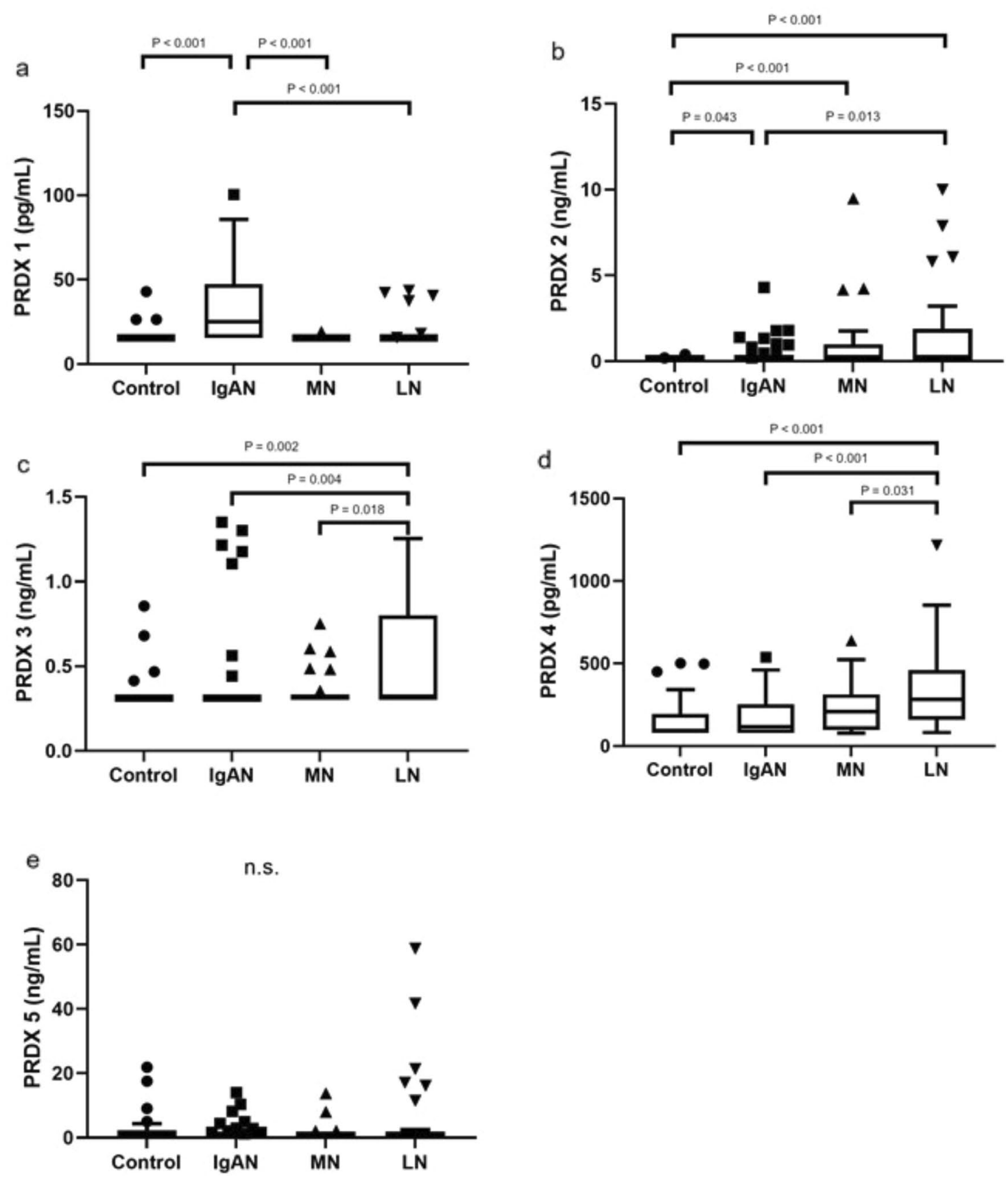

Fig. 2 Peroxiredoxin (PRDX) 1-5 concentrations in IgA nephropathy (IgAN), membranous nephropathy (MN), lupus nephritis (LN) and healthy controls. Data are presented as box-and-whisker plots; box represents interquartile range (IRQ) with line set as median value for each PRDX concentration, ends of whiskers represent $\pm 1.5 \mathrm{IQR}$

a clear tendency for higher concentrations of these PRDXs in GN patients has been observed in case of PRDX 3.

\section{Subclass Variability}

Depending on the PRDX subclass, the serum concentration varied between $\mathrm{IgAN}, \mathrm{MN}$, and $\mathrm{LN}$ patients and healthy

of value (maximum/minimum), and individual data points indicate outliers (Mann-Whitney $U$ test). $P$ value was considered significant if $<0.05$ and $<0.033$ after Bonferroni correction; (a) PRDX 1; (b) PRDX 2; (c) PRDX 3; (d) PRDX 4; (e) PRDX 5; n.s. not significant

controls (Fig. 2). IgAN patients had significantly higher concentrations of PRDX 1 and $2(P<0.001$ and $<0.043$, respectively; however, only PRDX 1 remained significant after Bonferroni correction; Fig. 2a, b); the MN group had almost the same PRDX levels, except for PRDX $2(P=0.001$; Fig. 2b), whereas the levels of PRDX 2, 3, and 4 in LN patients were significantly elevated $(P<0.001,<0.002$, and 
$<0.001$, respectively; Fig. $2 b-$ d) compared to those in the control group. Importantly, we noticed significant differences between the disease groups. Comparing IgAN and MN, higher PRDX 1 level was revealed level in IgAN patients (Fig. 2a). The significant differences between IgAN and LN varied depending on the PRDX subclass (1-4) (Fig. 2a-d), whereas comparing $\mathrm{LN}$ assessment and $\mathrm{MN}$ revealed higher levels of PRDX 3 and 4 in LN individuals (Fig. 2c, d).

To confirm the diagnostic accuracy of PRDX levels, we performed ROC analysis to strengthen the significance of the obtained results. The most prominent AUC values were detected for PRDX 2 (0.652) and 4 (0.653) to discriminate GN from controls and for PRDX 1 to discriminate IgAN from LN (0.733) and MN (0.788) (Table 2).

\section{Single-Patient PRDX Concentration Panel (Heatmap)}

Based on our results, we prepared a heatmap (Fig. 3) of PRDX concentrations for all the studied groups. The detection range was set as consecutive concentrations obtained from the standard curve (Table 3). The heatmap suggests the PRDX 1 potential to differentiate IgAN from other GN or controls. Moreover, PRDX 2 concentrations seem significantly higher in LN patients, than in IgAN and controls (as shown in Fig. 2b). This illustrates possible differential GN diagnostic pattern for PRDX 1 and 2; however, to confirm it is applicability, further studies addressing the subclass variability, relationship to such parameters as age, eGFR, proteinuria and anemia are required.

\section{Correlations}

\section{Glomerular Filtration Rate}

We observed significant correlation between PRDX 2 serum concentration and renal function as expressed by the eGFR in $\operatorname{IgAN}(P=0.001)$ and $\mathrm{LN}(P=0.001)$ patients (Fig. 4a, b), and similarly for PRDX 3 in MN $(P=0.041)$ and IgAN $(P=0.041)$ patients (Fig. $4 \mathrm{c}, \mathrm{d})$.

\section{Complement components C3 and C4}

The complement component concentration showed an inverse association with PRDX 1 in $\operatorname{IgAN}(P=0.031)$ and LN $(P=0.005$ and $P=0.008)$ patients (Fig. 5a, b, c) and PRDX $3(P=0.032$ and $P=0.035)$ in $\mathrm{LN}$ patients (Fig. $5 \mathrm{~d}, \mathrm{e})$. An association was also found with PRDX 3 in the whole GN group (Supplementary Table 1) (Fig. 5).

\section{Other Parameters}

In addition, a significant inverse correlation between PRDX 2 and hematocrit (HCT) was observed in the GN group
$(P=0.001)$. Further analysis revealed that HCT was correlated with PRDX 2 in LN only $(P=0.011)$. The association between hemoglobin (HGB) level and serum PRDX 2 concentration was found in the whole GN group $(P=0.001)$ and in $\operatorname{IgAN}(P=0.025)$ and LN $(P=0.005)$ separately (Supplementary Table 1$)$.

We noticed a significant correlation between PRDX 3 and $24 \mathrm{~h}$ proteinuria in the whole $\mathrm{GN}$ group $(P=0.013)$, but not in IgAN, $\mathrm{MN}$ or $\mathrm{LN}$ separately. Moreover, $24 \mathrm{~h}$ proteinuria was correlated to PRDX 2 only in MN patients $(P=0.014)$ (Supplementary Table 1). At the same time, we did observe an association of PRDX with serum proteinogram changes. For example, in IgAN patients, serum $\alpha-1$ and $\beta-1$ globulins, were correlated significantly with PRDX $1(P=0.032$; $P=0.001$, respectively), while $\beta-2$ globulins were correlated with PRDX $1(P=0.021), 3(P=0.018)$ and $4(P=0.004)$. Furthermore, in the MN group, $\beta-2$ globulins correlated with PRDX $2(P=0.033)$, and in the LN group, $\beta-1$ globulins correlated with PRDX $1(P=0.048)$ (Supplementary Table 1).

A significant correlation was also found between BMI and PRDX 5 in $\operatorname{IgAN}$ patients $(P=0.012)$. Other significant associations are summarized in Supplementary Table 1.

\section{Discussion}

In this study, we show that serum levels of PRDX 1-4 were significantly elevated in IgAN, MN, and LN patients compared to healthy individuals. In addition to the differences between disease and control groups, we also found that each GN type revealed a distinct PRDX pattern. There is no straight explanation why there would be differences in oxidative stress markers between different types of GN. The data on biological reasons for these differences are largely missing.

Oxidative stress is defined as a disturbance in the natural ability of cells to maintain a balance between pro- and antioxidant systems (Krata et al. 2018; Selvaskandan et al. 2020; Sethi et al. 2019, 2020; Yanagawa et al. 2014). Although moderate levels of hydrogen peroxide are necessary for numerous cellular processes, in excessive concentrations it can cause cell and tissue damage (mediated by oxidation of lipids, proteins, or DNA). Several mechanisms are responsible for the removal of reactive oxygen species, including superoxide dismutase, catalase, peroxidase, the peroxide-redox-thioredoxin-thioredoxin reductase enzymatic chain, and a number of non-enzymatic antioxidants (Descamps-Latscha et al. 2001). PRDXs are known as biomarkers for cancer (Basu et al. 2011; O'Leary et al. 2014), bacterial infections (Yang et al. 2018), and neurodegenerative (Goemaere and Knoops 2012) and inflammatory-related diseases (Park et al. 2016). Their role in the pathophysiology and/or diagnostics of IgAN, MN, and LN has not been characterized yet. 


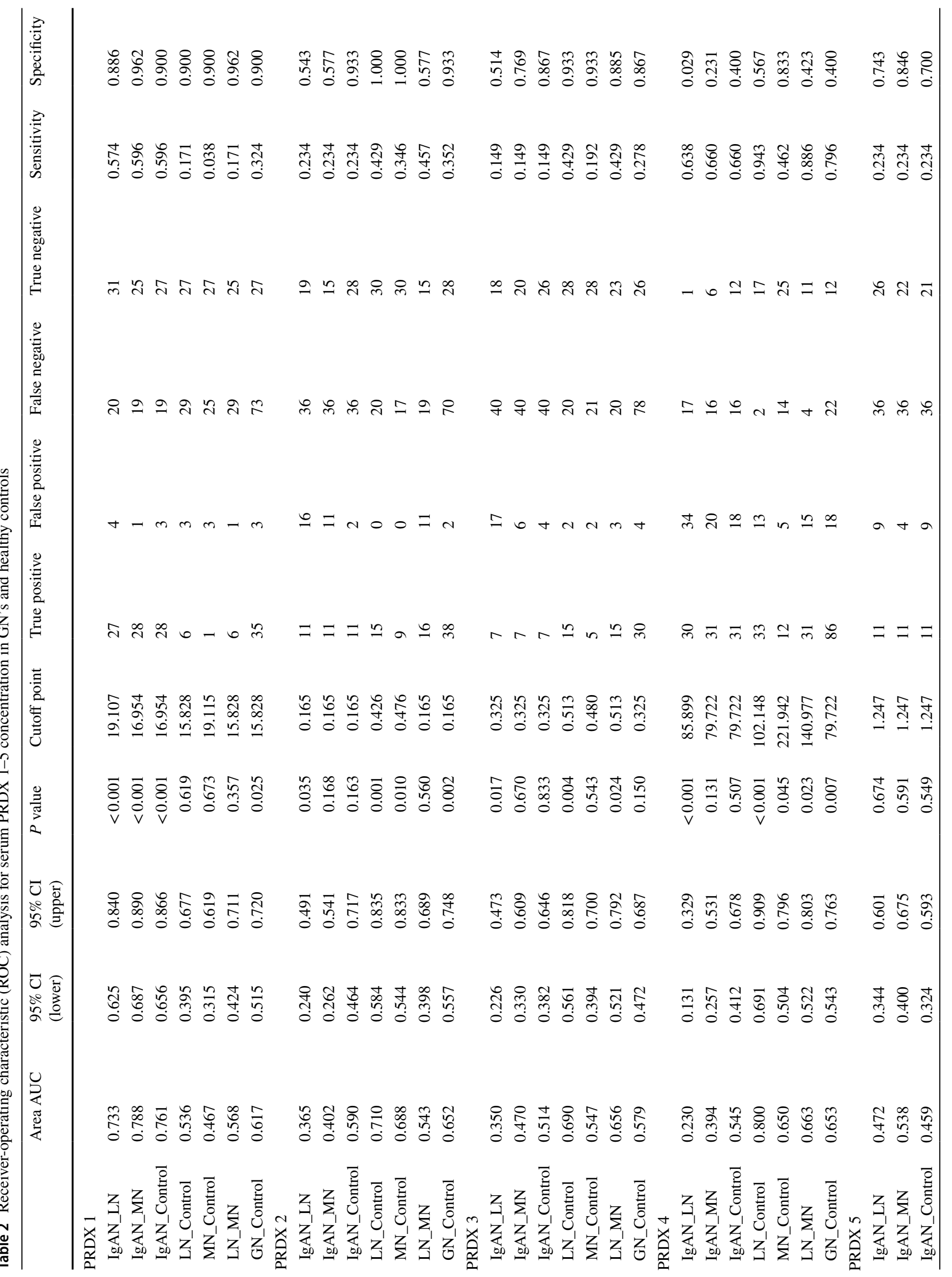




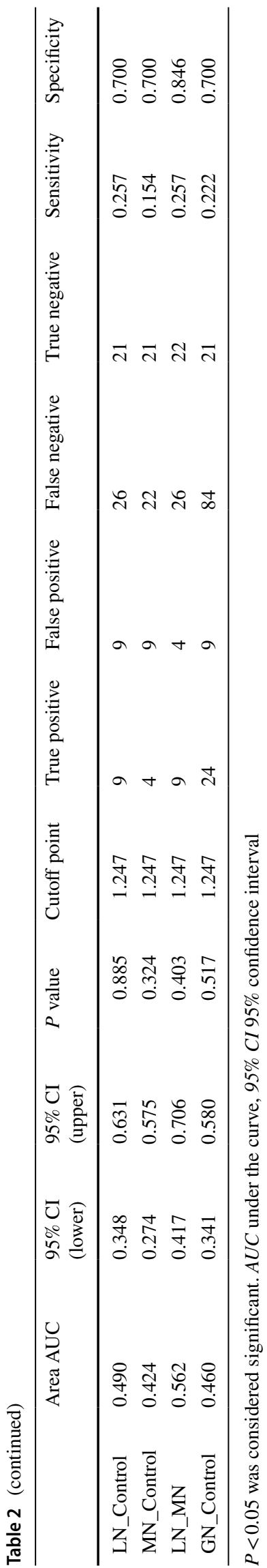

The fundamental method of following up CKD, including GN patients, is eGFR estimation. We observed an inverse correlation between eGFR and PRDX 2 in IgAN and LN but not MN patients and PRDX3 in IgAN and MN individuals. For the obvious reasons, a simple link between eGFR and serum PRDX probably does not exist. However, declining eGFR is the evident effect of kidney disease progression involving, e.g., oxidative stress. Coexisting secondary anemia or other comorbidities could additionally influence PRDX levels in serum (discussed below). Another gold standard for GN follow-up is 24-h urine protein loss analysis. Higher proteinuria usually indicates more advanced or active GN. However, we observed only one correlation between PRDX 2 and $24 \mathrm{~h}$ proteinuria in MN patients. The implication of this is unclear. The highly variable degree of proteinuria in $\mathrm{MN}$ patients, related to the $\mathrm{MN}$ biology could explain this finding. On the other hand, a potential relation to protein loss may be suspected also in other GNs based on the observed PRDX association with serum proteinogram changes. Therefore, the causes of proteinogram changes are probably multifactorial and OS is differentially involved. Moreover, to date, there has not been much published data about PRDX and GN, particularly in the context of proteinuria. Of note, proteinuria may additionally serve as an indirect indicator of the GN activity. One could speculate that due to the relative unspecificity of OS, PRDX serum levels might be expected to reflect disease activity rather than disease itself. This speculation could not be elucidated with our study design, since each studied GN has distinct activity scale. Thus, the comparison of disease activity between these groups is impossible. Moreover, diagnostic renal biopsies were performed in history, not at the time of sampling.

OS is implicated in various or almost all disease states, including atherosclerosis, cardiovascular disease, obesity, diabetes, cancer, neurodegeneration, aging, drugs (e.g., allopurinol) and many others. Such conditions could confound the results and anemia belongs to the most potent OS confounders. Along with CKD progression, the uremic toxins increase red blood cell (RBC) damage and erythropoietin deficiency reduces HGB production, resulting in tissue hypoxia. The latter may stimulate OS responses, including, e.g., increased PRDXs (Gwozdzinski et al. 2021). Indeed, we found a correlation between HGB and serum PRDX 2 concentrations in IgAN and LN patients. It was reported that PRDX 2 plays an important role in the protection of RBCs from OS through HGB autoxidation (Johnson et al. 2005), e.g., in iron-deficiency anemia (Nagababu et al. 2008). The protective function of PRDX 2 in HGB stability has been investigated in PRDX 2 knockout mice and RBCs from patients with hereditary hemolytic anemia. The authors of this study suggested that PRDX 2 could bind to HGB and protect it from oxidative denaturation and aggregation in RBCs (Han et al. 2012). 
Fig. 3 PRDX concentration panel (heatmap) of study participants

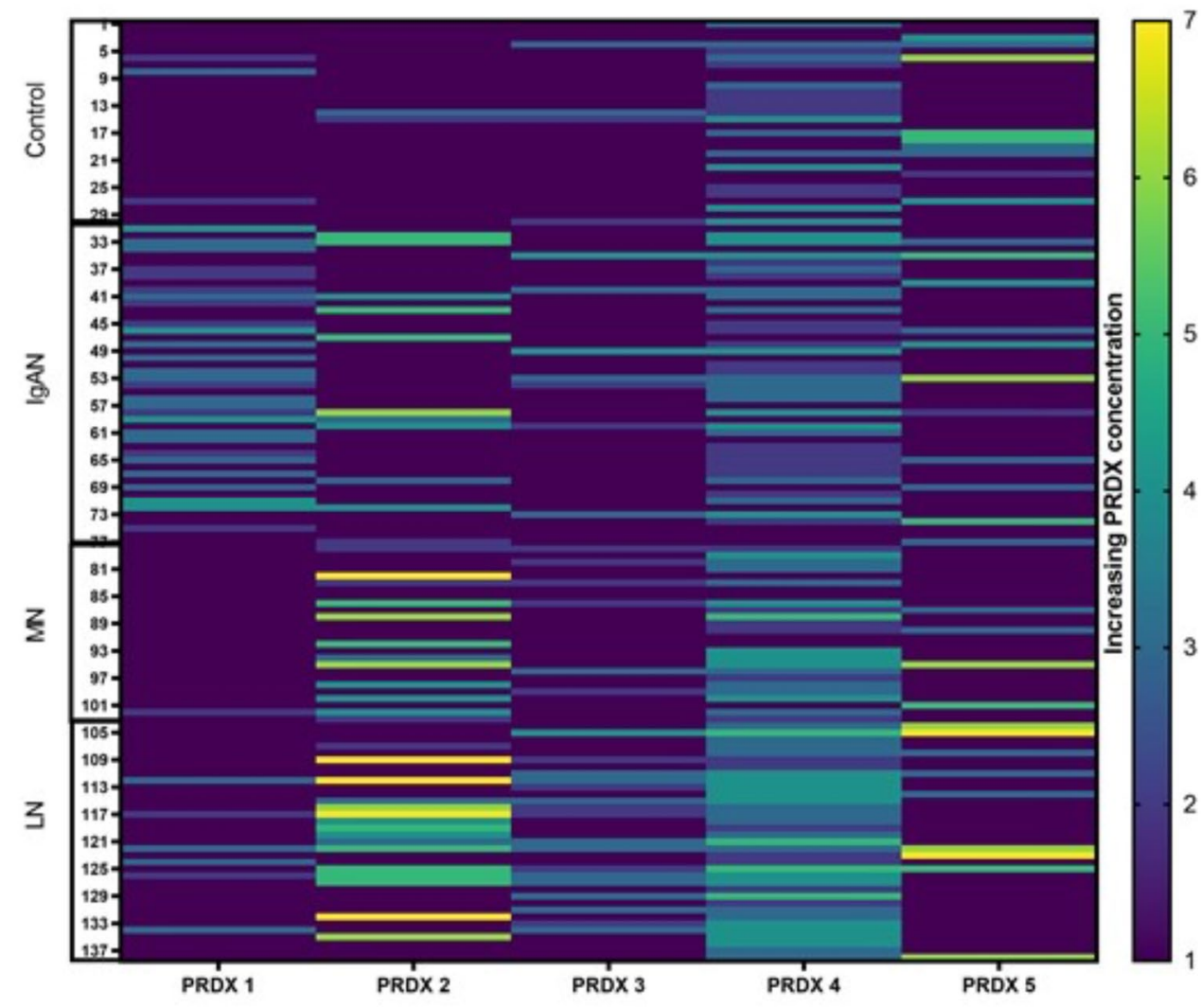

Table 3 Consecutive standard PRDX dilutions obtained from standard curve

\begin{tabular}{llllll}
\hline $\begin{array}{l}\text { PRDX } \\
\text { Concentration range }\end{array}$ & PRDX 1 $(\mathrm{pg} / \mathrm{mL})$ & PRDX 2 $(\mathrm{ng} / \mathrm{mL})$ & PRDX 3 $(\mathrm{ng} / \mathrm{mL})$ & PRDX 4 (pg/mL) & PRDX 5 $(\mathrm{ng} / \mathrm{mL})$ \\
\hline 1 & & & & $<$ \\
2 & $<15.6$ & $<0.156$ & $<0.312$ & $<78.0$ & $0.78-1.56$ \\
3 & $15.6-31.2$ & $0.156-0.312$ & $0.312-0.625$ & $78.0-156$ & $1.57-3.12$ \\
4 & $31.3-62.5$ & $0.313-0.625$ & $0.626-1.25$ & $157-312$ & $3.13-6.25$ \\
5 & $62.6-125$ & $0.626-1.25$ & $1.251-2.5$ & $313-625$ & $6.26-12.5$ \\
6 & $125.1-250$ & $1.251-2.5$ & $2.501-5.0$ & $626-1250$ & $12.51-25$ \\
7 & $250.1-500$ & $2.501-5.0$ & $5.001-10$ & $1251-2500$ & $25.01-50$ \\
\hline
\end{tabular}

What is very important in our study is the fact that our patients had stage 2 CKD and an average eGFR of 71, 74, and $84 \mathrm{~mL} / \mathrm{min}$ (for IgAN, MN, and LN, respectively), thus had normal RBC and HGB levels. One of the limitations of our study was relatively low patient numbers in each GN group, which precluded analysis of the effect other comorbidities on PRDX concentrations. Therefore, the question is, what does the increased serum PRDX really mean? Do PRDX levels behave as a damage marker or damage type marker? Is it possible that it is just a very early marker of preexisting hypoxia and/or OS in CKD patients long before the development of even early stages of anemia? The fact that different GNs enhance different PRDX classes also suggests different disease-specific mechanisms of hypoxia, which needs additional research. Another question is whether the serum concentrations of PRDXs should be only used as markers for a specific disease type or can also reflects the activity of this particular disease at the time of sample collection? In addition, oxidative stress activity in serum may not always reflect that of changes cellular microdomains in the kidney, which should be taken under consideration while interpreting the results of the current study. Finally, it is also worth adding that evaluation of all GN groups vs. healthy individuals 

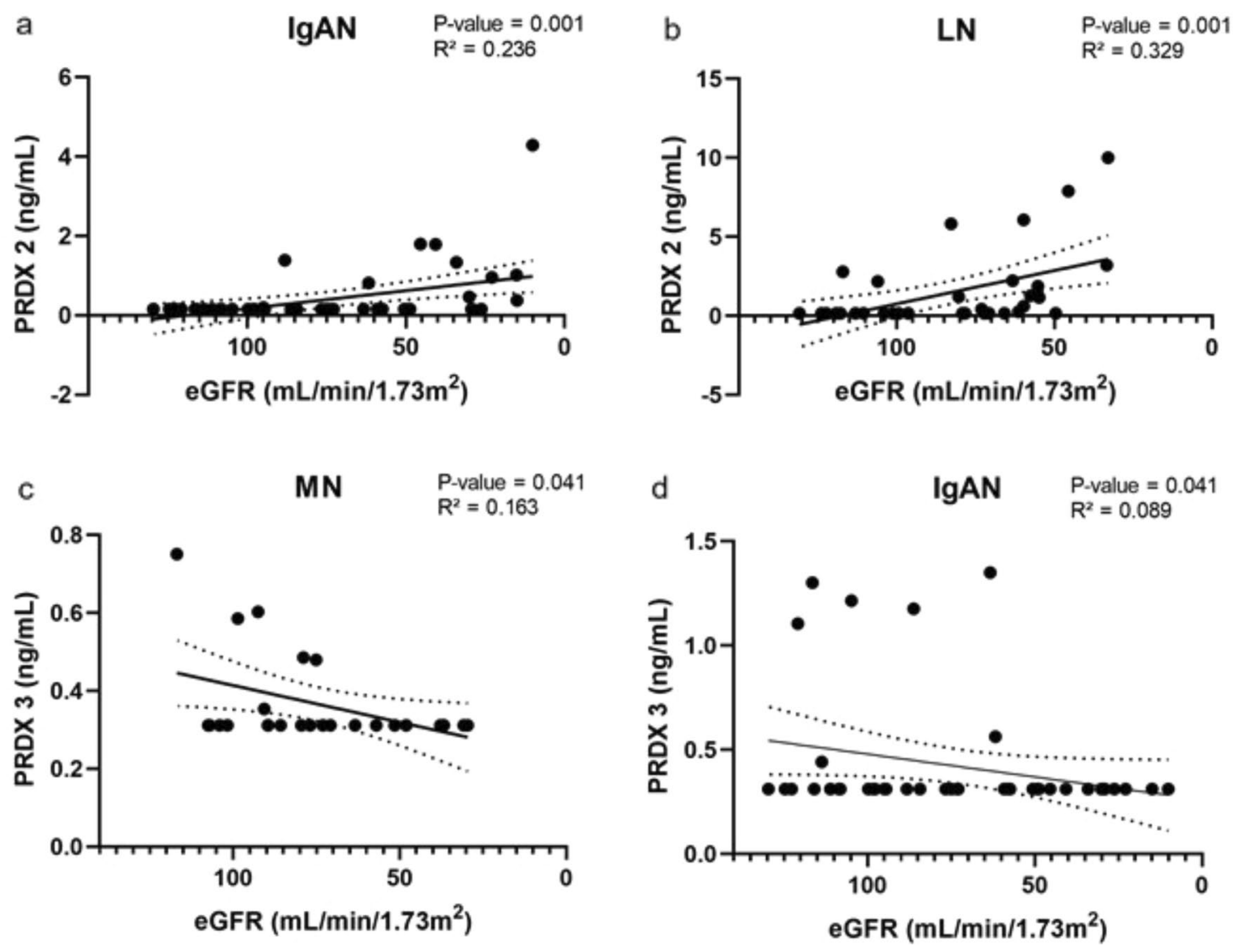

Fig. 4 Spearman's correlation analysis of eGFR and PRDX 2 (IgAN, LN) and PRDX 3 in (IgAN, MN) patients. Correlation was fitted with linear model with $95 \%$ confidence interval bands; $P<0.05$ was considered significant

showed significant differences in PRDX 1 and 2 (as illustrated on a heatmap). Therefore, it is an open question whether serum PRDX 1 and 2 might help in the future to distinguish patients suspected of having IgAN, MN-, or LN-related oxidative stress?

Complement activation and its regulation are complex phenomena. It is not definitive whether these phenomena are the direct primary cause or the effector mechanism of kidney injury, but they are involved in GN development and progression; thus, inhibiting them has become an emerging treatment option (Kaartinen et al. 2019). In patients with IgAN, the immunoglobulin A deposited in the mesangial area of the kidney can activate the complement system through either the lectin pathway or an alternative pathway. This may amplify the local inflammatory response and contribute to renal injury (Daha and van Kooten 2016). The immunopathology of human MN shows evidence of complement activation within immune deposits (Beck and Salant 2010). Therefore, it is thought likely that the complement system also plays a substantial role in MN pathogenesis. Although serum levels of complement proteins are usually normal in MN patients, it was recently reported that measuring the circulating complement activation products may be a way to detect ongoing complement activation (Zhang et al. 2019). Complement also has an important role in the pathogenesis of LN. On the one hand, a deficiency of complement components predisposes to lupus, while on the other hand, excess complement activation increases renal damage, and measuring it is done to assess disease activity (Sharma et al. 2020). Although OS involvement in renal injury being driven by complement activation seems obvious, to the best of our knowledge, to date there are no available data linking complement activation in GNs to PRDXs. We assume that the disease-specific PRDX associations with circulating $\mathrm{C} 3$ and $\mathrm{C} 4$ complement components observed in our study suggest differential OS responses depending on the GN etiology and the mode of complement activation. If so, PRDX subclass assessment might be useful as an adjunct in the diagnosis of GNs.

Another finding of our study is the positive correlation between PRDX 5 and BMI in the IgAN group. It was previously demonstrated in obese mouse models that PRDX 5 inhibits adipogenesis by modulating ROS generation and adipogenic gene expression, implying that it may serve as a potential target to prevent and treat obesity (Kim et al. 2018). Moreover, in vitro and in vivo experiments 

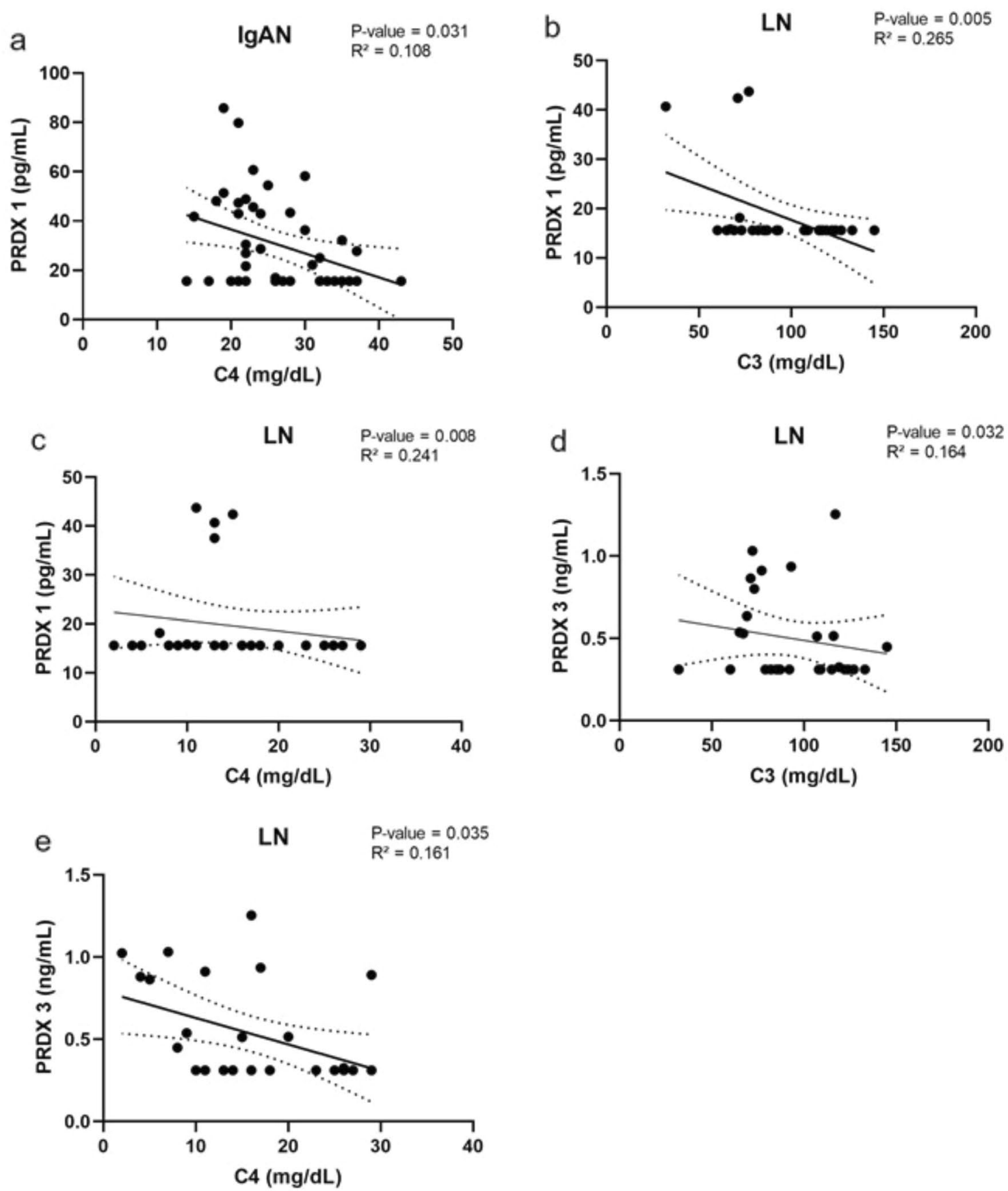

Fig. 5 Spearman's correlation analysis of complements C3 and C4 in IgAN and LN patients. Correlation was fitted with linear model with 95\% confidence interval, $P<0.05$ was considered significant

suggested that PRDX 5 functions as a protective regulator in fatty liver disease and may be a valuable therapeutic target for the management of obesity-related metabolic diseases (Kim et al. 2020). Taking these findings and our results together, it is possible that PRDX 5 is upregulated in response to chronic nephritis depending on BMI value, but this hypothesis needs further elucidation.
It is important to mention that in the current study the serum levels of PRDXs were evaluated, which might not reflect the intracellular changes in PRDXs expression within the tissue. As PRDXs can be released as damage-associated molecular pattern markers from injured tissues (He et al. 2019), this subject warrants further investigations. 
Generally, our study indicates potential applicability of antioxidant supplementation in renal disease. The potential compounds to be used are, for instance: coenzyme Q10, Vitamins B, C, D, and E, L-carnitine, statins, or $\mathrm{N}$-acetylcysteine (Liakopoulos et al. 2019). Indeed, application of coenzyme Q10 has been reported beneficial in the treatment of chronic diseases, including CKD (Gutierrez-Mariscal et al. 2020).

In conclusion, our results highlight the link between PRDXs and GNs (IgAN, MN, and LN). Our study indicates that individual PRDXs can play roles in pathophysiology of selected GNs and that their concentrations in serum may become useful as new supplementary diagnostic markers in IgAN, MN, and LN. Validation studies including other kidney diseases are required to better understand GNs and enable their less invasive diagnosis.

Supplementary Information The online version contains supplementary material available at https://doi.org/10.1007/s00005-021-00638-1.

Acknowledgements This project is the continuation of our study on the role of 2-Cys-PRDXs in GNs, the results of which have been included in the patent application from February 2017 to the European Patent Office (No. WO/2018/141975). The latest letter received from the patent office, dated 17 April 2020, stated: "The claimed subject matter is considered novel over the cited prior Art. 54 EPC". We thank prof. Krzysztof Kiryluk from the Division of Nephrology in the Department of Medicine at Columbia University (New York, USA) for consulting the results and the final version of the manuscript. The routine laboratory tests were performed at the diagnostic laboratory of Infant Jesus Hospital, University Medical Center of the Medical University of Warsaw, during routine patient visits to the Nephrology and Transplantation Outpatient Clinic. The healthy control subjects donated their blood voluntarily. The ELISA measurements were performed with kind support from Kamila Gala, PhD. Figures were prepared with GraphPad Prism 9 software.

Author Contributions KM and NK designed the study; NK carried out the experiments; NK and KM analyzed the data and generated the figures; $\mathrm{BF}, \mathrm{BM}$, and $\mathrm{KM}$ collected samples; $\mathrm{NK}, \mathrm{BF}, \mathrm{RZ}, \mathrm{BM}, \mathrm{MZ}$, LP, and KM analyzed the data; NK, BF, RZ, BM, LP, and KM drafted and revised the article; all the authors approved the final version of the manuscript.

Funding This research was supported by the Medical University of Warsaw, internal funding No. N1/16/16.

Data Availability Data available upon request.

Code Availability Not applicable.

\section{Declarations}

Conflict of Interest None declared.

Ethics Approval The study was conducted according to the guidelines of the Declaration of Helsinki for research on human subjects of 1975, revised in 2013, and approved by the Ethics Committee of the Medical University of Warsaw (KB/9/2010 and KB/199/2016).

Consent to Participate Informed consent was obtained from all the subjects involved in the study.

Consent for Publication Not applicable.

Open Access This article is licensed under a Creative Commons Attribution 4.0 International License, which permits use, sharing, adaptation, distribution and reproduction in any medium or format, as long as you give appropriate credit to the original author(s) and the source, provide a link to the Creative Commons licence, and indicate if changes were made. The images or other third party material in this article are included in the article's Creative Commons licence, unless indicated otherwise in a credit line to the material. If material is not included in the article's Creative Commons licence and your intended use is not permitted by statutory regulation or exceeds the permitted use, you will need to obtain permission directly from the copyright holder. To view a copy of this licence, visit http://creativecommons.org/licenses/by/4.0/.

\section{References}

Adimora NJ, Jones DP, Kemp ML (2010) A model of redox kinetics implicates the thiol proteome in cellular hydrogen peroxide responses. Antioxid Redox Signal 13:731-743. https://doi.org/10. 1089/ars.2009.2968

Aranda-Rivera AK, Cruz-Gregorio A, Aparicio-Trejo OE et al (2021) Mitochondrial redox signaling and oxidative stress in kidney diseases. Biomolecules 11:1144. https://doi.org/10.3390/biom1 1081144

Basu A, Banerjee H, Rojas H et al (2011) Differential expression of peroxiredoxins in prostate cancer: consistent upregulation of PRDX3 and PRDX4. Prostate 71:755-765. https://doi.org/10. 1002/pros.21292

Beck LH Jr, Salant DJ (2010) Membranous nephropathy: recent travels and new roads ahead. Kidney Int 77:765-770. https://doi.org/10. 1038/ki.2010.34

Brück K, Stel VS, Gambaro G et al (2016) CKD prevalence varies across the European general population. J Am Soc Nephrol 27:2135-2147. https://doi.org/10.1681/asn.2015050542

Cachofeiro V, Goicochea M, de Vinuesa SC et al (2008) Oxidative stress and inflammation, a link between chronic kidney disease and cardiovascular disease. Kidney Int Suppl 111:S4-9. https:// doi.org/10.1038/ki.2008.516

Daenen K, Andries A, Mekahli D et al (2019) Oxidative stress in chronic kidney disease. Pediatr Nephrol 34:975-991. https://doi. org/10.1007/s00467-018-4005-4

Daha MR, van Kooten C (2016) Role of complement in IgA nephropathy. J Nephrol 29:1-4. https://doi.org/10.1007/s40620-015-0245-6

Descamps-Latscha B, Drüeke T, Witko-Sarsat V (2001) Dialysisinduced oxidative stress: biological aspects, clinical consequences, and therapy. Semin Dial 14:193-199. https://doi.org/ 10.1046/j.1525-139x.2001.00052.x

Floege J, Amann K (2016) Primary glomerulonephritides. Lancet 387:2036-2048. https://doi.org/10.1016/s0140-6736(16) 00272-5

Foreman KJ, Marquez N, Dolgert A et al (2018) Forecasting life expectancy, years of life lost, and all-cause and cause-specific mortality for 250 causes of death: reference and alternative scenarios for 2016-40 for 195 countries and territories. 
Lancet 392:2052-2090. https://doi.org/10.1016/s0140-6736(18) 31694-5

Gao J, Meyer K, Borucki K et al (2018) Multiplex immuno-MALDITOF MS for targeted quantification of protein biomarkers and their proteoforms related to inflammation and renal dysfunction. Anal Chem 90:3366-3373. https://doi.org/10.1021/acs. analchem.7b04975

Goemaere J, Knoops B (2012) Peroxiredoxin distribution in the mouse brain with emphasis on neuronal populations affected in neurodegenerative disorders. J Comp Neurol 520:258-280. https://doi.org/10.1002/cne.22689

Gutierrez-Mariscal FM, Arenas-de Larriva AP, Limia-Perez L et al (2020) Coenzyme Q(10) supplementation for the reduction of oxidative stress: clinical implications in the treatment of chronic diseases. Int J Mol Sci 21:7870. https://doi.org/10.3390/ijms2 1217870

Gwozdzinski K, Pieniazek A, Gwozdzinski L (2021) Reactive oxygen species and their involvement in red blood cell damage in chronic kidney disease. Oxid Med Cell Longev 2021:6639199. https://doi.org/10.1155/2021/6639199

Han YH, Kim SU, Kwon TH et al (2012) Peroxiredoxin II is essential for preventing hemolytic anemia from oxidative stress through maintaining hemoglobin stability. Biochem Biophys Res Commun 426:427-432. https://doi.org/10.1016/j.bbrc.2012.08.113

He Y, Li S, Tang D et al (2019) Circulating peroxiredoxin-1 is a novel damage-associated molecular pattern and aggravates acute liver injury via promoting inflammation. Free Radic Biol Med 137:24-36. https://doi.org/10.1016/j.freeradbiomed.2019.04. 012

Hill NR, Fatoba ST, Oke JL et al (2016) Global prevalence of chronic kidney disease - a systematic review and meta-analysis. PLoS ONE 11:e0158765. https://doi.org/10.1371/journal.pone.01587 65

Hwang I, Uddin MJ, Lee G et al (2019) Peroxiredoxin 3 deficiency accelerates chronic kidney injury in mice through interactions between macrophages and tubular epithelial cells. Free Radic Biol Med 131:162-172. https://doi.org/10.1016/j.freeradbio med.2018.12.002

Irazabal MV, Torres VE (2020) Reactive oxygen species and redox signaling in chronic kidney disease. Cells 9:1342. https://doi. org/10.3390/cells9061342

Jeong J, Kim Y, Kyung Seong J et al (2012) Comprehensive identification of novel post-translational modifications in cellular peroxiredoxin 6. Proteomics 12:1452-1462. https://doi.org/10. 1002/pmic.201100558

Johnson RM, Goyette G Jr, Ravindranath Y et al (2005) Hemoglobin autoxidation and regulation of endogenous $\mathrm{H} 2 \mathrm{O} 2$ levels in erythrocytes. Free Radic Biol Med 39:1407-1417. https://doi. org/10.1016/j.freeradbiomed.2005.07.002

Kaartinen K, Safa A, Kotha S et al (2019) Complement dysregulation in glomerulonephritis. Semin Immunol 45:101331. https://doi. org/10.1016/j.smim.2019.101331

Kim MH, Park SJ, Kim JH et al (2018) Peroxiredoxin 5 regulates adipogenesis-attenuating oxidative stress in obese mouse models induced by a high-fat diet. Free Radic Biol Med 123:27-38. https://doi.org/10.1016/j.freeradbiomed.2018.05.061

Kim MH, Seong JB, Huh JW et al (2020) Peroxiredoxin 5 ameliorates obesity-induced non-alcoholic fatty liver disease through the regulation of oxidative stress and AMP-activated protein kinase signaling. Redox Biol 28:101315. https://doi.org/10.1016/j.redox. 2019.101315

Krata N, Zagożdżon R, Foroncewicz B et al (2018) Oxidative stress in kidney diseases: the cause or the consequence? Arch Immunol Ther Exp 66:211-220. https://doi.org/10.1007/s00005-017-0496-0

Lee E, Lee HS (2018) Peroxidase expression is decreased by palmitate in cultured podocytes but increased in podocytes of advanced diabetic nephropathy. J Cell Physiol 233:9060-9069. https://doi. org/10.1002/jcp.26875

Liakopoulos V, Roumeliotis S, Bozikasb A et al (2019) Antioxidant supplementation in renal replacement therapy patients: is there evidence? Oxid Med Cell Longev 2019:9109473. https://doi.org/ 10.1155/2019/9109473

McGrogan A, Franssen CF, de Vries CS (2011) The incidence of primary glomerulonephritis worldwide: a systematic review of the literature. Nephrol Dial Transplant 26:414-430. https://doi.org/ 10.1093/ndt/gfq665

Moszczuk B, Kiryluk K, Pączek L et al (2021) Membranous nephropathy: from research bench to personalized care. J Clin Med 10:1205. https://doi.org/10.3390/jcm10061205

Mucha K, Bakun M, Jaźwiec R et al (2014) Complement components, proteolysis-related, and cell communication-related proteins detected in urine proteomics are associated with IgA nephropathy. Pol Arch Med Wewn 124:380-386. https://doi.org/10.20452/ pamw. 2345

Mucha K, Foroncewicz B, Pączek L (2016) How to diagnose and follow patients with glomerulonephritis without kidney biopsy? Pol Arch Med Wewn 126:471-473. https://doi.org/10.20452/pamw. 3510

Na W, Yi K, Song YS et al (2017) Dissecting the relationships of IgG subclasses and complements in membranous lupus nephritis and idiopathic membranous nephropathy. PLoS ONE 12:e0174501. https://doi.org/10.1371/journal.pone.0174501

Nagababu E, Gulyani S, Earley CJ et al (2008) Iron-deficiency anaemia enhances red blood cell oxidative stress. Free Radic Res 42:824 829. https://doi.org/10.1080/10715760802459879

O’Leary PC, Terrile M, Bajor M et al (2014) Peroxiredoxin-1 protects estrogen receptor $\alpha$ from oxidative stress-induced suppression and is a protein biomarker of favorable prognosis in breast cancer. Breast Cancer Res 16:R79. https://doi.org/10.1186/bcr3691

Pac M, Krata N, Moszczuk B, Wyczałkowska-Tomasik A, Kaleta B, Foroncewicz B, Rudnicki W, Pączek L, Mucha K (2021) NR3C1 Glucocorticoid Receptor Gene Polymorphisms Are Associated with Membranous and IgA Nephropathies. Cells 10(11):3186. https://doi.org/10.3390/cells10113186

Park MH, Jo M, Kim YR et al (2016) Roles of peroxiredoxins in cancer, neurodegenerative diseases and inflammatory diseases. Pharmacol Ther 163:1-23. https://doi.org/10.1016/j.pharmthera.2016.03.018

Patel M, Clarke AM, Bruce IN et al (2006) The prevalence and incidence of biopsy-proven lupus nephritis in the UK: Evidence of an ethnic gradient. Arthritis Rheum 54:2963-2969. https://doi. org/10.1002/art.22079

Perkins A, Nelson KJ, Parsonage D et al (2015) Peroxiredoxins: guardians against oxidative stress and modulators of peroxide signaling. Trends Biochem Sci 40:435-445. https://doi.org/10.1016/j.tibs. 2015.05.001

Pippias M, Kramer A, Noordzij M et al (2017) The European renal association-European dialysis and transplant association registry annual report 2014: a summary. Clin Kidney J 10:154-169. https://doi.org/10.1093/ckj/sfw135

Placzek WJ, Yanagawa H, Makita Y et al (2018) Serum galactosedeficient-IgA1 and IgG autoantibodies correlate in patients with IgA nephropathy. PLoS ONE 13:e0190967. https://doi.org/10. 1371/journal.pone.0190967

Selvaskandan H, Shi S, Twaij S et al (2020) Monitoring immune responses in IgA nephropathy: biomarkers to guide management. Front Immunol 11:572754. https://doi.org/10.3389/fimmu.2020. 572754

Sethi S, Madden BJ, Debiec H et al (2019) Exostosin 1/exostosin 2-associated membranous nephropathy. J Am Soc Nephrol 30:1123-1136. https://doi.org/10.1681/asn.2018080852

Sethi S, Debiec H, Madden B et al (2020) Neural epidermal growth factor-like 1 protein (NELL-1) associated membranous nephropathy. 
Kidney Int 97:163-174. https://doi.org/10.1016/j.kint.2019.09. 014

Sharapov MG, Goncharov RG, Filkov GI et al (2020) Comparative study of protective action of exogenous 2-Cys peroxiredoxins (Prx1 and Prx2) under renal ischemia-reperfusion injury. Antioxidants 9:680. https://doi.org/10.3390/antiox9080680

Sharma M, Vignesh P, Tiewsoh K et al (2020) Revisiting the complement system in systemic lupus erythematosus. Expert Rev Clin Immunol 16:397-408. https://doi.org/10.1080/1744666x.2020. 1745063

Vassalotti JA, Fox CH, Becker BN (2010) Risk factors and screening for chronic kidney disease. Adv Chronic Kidney Dis 17:237-245. https://doi.org/10.1053/j.ackd.2010.03.003

Winterbourn CC (2008) Reconciling the chemistry and biology of reactive oxygen species. Nat Chem Biol 4:278-286. https://doi.org/ $10.1038 /$ nchembio. 85

Wu CL, Su TC, Chang CC et al (2017) Tubular peroxiredoxin 3 as a predictor of renal recovery from acute tubular necrosis in patients with chronic kidney disease. Sci Rep 7:43589. https://doi.org/10. 1038/srep43589

Xie J, Liu L, Mladkova N, Li Y, Ren H, Wang W, Cui Z, Lin L, Hu X, Yu X, Xu J, Liu G, Caliskan Y, Sidore C, Balderes O, Rosen RJ, Bodria M, Zanoni F, Zhang JY, Krithivasan P, Mehl K, Marasa M, Khan A, Ozay F, Canetta PA, Bomback AS, Appel GB, SannaCherchi S, Sampson MG, Mariani LH, Perkowska-Ptasinska A, Durlik M, Mucha K, Moszczuk B, Foroncewicz B, Pączek L, Habura I, Ars E, Ballarin J, Mani LY, Vogt B, Ozturk S, Yildiz A, Seyahi N, Arikan H, Koc M, Basturk T, Karahan G, Akgul SU, Sever MS, Zhang D, Santoro D, Bonomini M, Londrino F, Gesualdo L, Reiterova J, Tesar V, Izzi C, Savoldi S, Spotti D, Marcantoni C, Messa P, Galliani M, Roccatello D, Granata S, Zaza G, Lugani F, Ghiggeri G, Pisani I, Allegri L, Sprangers B, Park JH, Cho B, Kim YS, Kim DK, Suzuki H, Amoroso A, Cattran DC, Fervenza FC, Pani A, Hamilton P, Harris S, Gupta S, Cheshire C,
Dufek S, Issler N, Pepper RJ, Connolly J, Powis S, Bockenhauer D, Stanescu HC, Ashman N, Loos RJF, Kenny EE, Wuttke M, Eckardt KU, Köttgen A, Hofstra JM, Coenen MJH, Kiemeney LA, Akilesh S, Kretzler M, Beck LH, Stengel B, Debiec H, Ronco P, Wetzels JFM, Zoledziewska M, Cucca F, Ionita-Laza I, Lee H, Hoxha E, Stahl RAK, Brenchley P, Scolari F, Zhao MH, Gharavi AG, Kleta R, Chen N, Kiryluk K (2020) The genetic architecture of membranous nephropathy and its potential to improve non-invasive diagnosis. Nat Commun. https://doi.org/10.1038/ s41467-020-15383-w

Yanagawa H, Suzuki H, Suzuki Y et al (2014) A panel of serum biomarkers differentiates IgA nephropathy from other renal diseases. PLoS ONE 9:e98081. https://doi.org/10.1371/journal.pone.00980 81

Yang HY, Lee TH (2015) Antioxidant enzymes as redox-based biomarkers: a brief review. BMB Rep 48:200-208. https://doi.org/ 10.5483/bmbrep.2015.48.4.274

Yang YZ, Zhao Y, Yang L et al (2018) Characterization of 2-Cys peroxiredoxin 3 and 4 in common carp and the immune response against bacterial infection. Comp Biochem Physiol B Biochem Mol Biol 217:60-69. https://doi.org/10.1016/j.cbpb.2017.12.012

Zacchia M, Marchese E, Trani EM et al (2020) Proteomics and metabolomics studies exploring the pathophysiology of renal dysfunction in autosomal dominant polycystic kidney disease and other ciliopathies. Nephrol Dial Transplant 35:1853-1861. https://doi. org/10.1093/ndt/gfz121

Zhang MF, Huang J, Zhang YM et al (2019) Complement activation products in the circulation and urine of primary membranous nephropathy. BMC Nephrol 20:313. https://doi.org/10.1186/ s12882-019-1509-5

Publisher's Note Springer Nature remains neutral with regard to jurisdictional claims in published maps and institutional affiliations. 